\title{
Ephemeride des periodischen Kometen Winnecke für die Erscheinung 1915.
}

(Fortsetzung der Ephemeride in A. N. 4787.)

Da die letzten Orte in A. N. 4787 durch ein kleines Versehen etwas entstellt erscheinen, so beginnt die nachstehende Fortsetzung mit diesen verbesserten Orten. $\mathbf{I}^{\mathrm{h}} \mathrm{m}$. Z. Berlin.

\begin{tabular}{|c|c|c|c|c|c|c|c|c|c|c|c|c|c|c|c|c|c|c|c|}
\hline \multirow{2}{*}{\multicolumn{2}{|c|}{$\begin{array}{c}\text { 191 } 5 \\
\text { Aug. } 22\end{array}$}} & \multicolumn{3}{|c|}{$\alpha$ app. } & \multicolumn{3}{|c|}{$\delta$ app. } & $\log r$ & $\log \Delta$ & \multicolumn{2}{|c|}{1915} & \multicolumn{3}{|c|}{$\alpha$ app. } & \multicolumn{3}{|c|}{$\delta$ app. } & \multirow[t]{2}{*}{$\log r$} & \multirow[t]{2}{*}{$\log \Delta$} \\
\hline & & I $3^{\mathrm{h}}$ & $32^{m}$ & ${ }^{m} 41 \div 36$ & $-3^{\circ}$ & $3^{\circ} 48^{\prime}$ & $57 " 5$ & 9.991928 & $0.05^{2} 34^{8}$ & Okt. & 4 & $x 6^{h}$ & $47^{\mathrm{n}}$ & $\mathrm{m}_{4} \mathrm{I}^{\mathrm{s}} \cdot 7 \mathrm{I}$ & $-33^{\circ}$ & ${ }^{\circ} 7^{\prime}$ & 26.0 & & \\
\hline & 23 & & 36 & 9.47 & 4 & 30 & I 8.0 & & & & 5 & & 53 & 30.67 & 33 & $3^{6}$ & I 3.8 & 0.0378 I 5 & 0.01478 \\
\hline & 24 & & 39 & 39.80 & 5 & I I & 58.6 & & & & 6 & I 6 & 59 & 22.40 & 34 & 3 & $5^{2.6}$ & & \\
\hline & 25 & & 43 & $\mathrm{I} 2.4 \mathrm{I}$ & 5 & 53 & 58.3 & & & & 7 & $\mathrm{I} 7$ & 5 & 16.70 & 34 & 30 & 20.8 & & \\
\hline & 26 & & 46 & $47 \cdot 37$ & 6 & 36 & 16.3 & 9.989012 & $0.043^{6} 44$ & & 8 & & 11 & I $3.3^{8}$ & 34 & 55 & 36.7 & & \\
\hline & 27 & & $5^{\circ}$ & 24.74 & 7 & I 8 & $5 \mathrm{I} .7$ & & & & 9 & & 17 & I $2.2 \mathrm{I}$ & 35 & I9 & 39.0 & 0.048221 & 0.0207 \\
\hline & 28 & & 54 & $4 \cdot 57$ & 8 & $I$ & $43 \cdot 5$ & & & & IO & & 23 & I 2.96 & 35 & 42 & 26.4 & & \\
\hline & 29 & I 3 & 57 & 46.90 & 8 & 44 & 50.6 & & & & I I & & 29 & $15 \cdot 38$ & $3^{6}$ & 3 & 57.8 & & \\
\hline & 30 & I 4 & I & 3 I.82 & 9 & 28 & 12.0 & 9.987620 & 0.035442 & & $\mathrm{r} 2$ & & 35 & $19.2 \mathrm{I}$ & $3^{6}$ & 24 & I 2.4 & & \\
\hline & $3^{I}$ & & 5 & 19.42 & IO & I I & 46.7 & & & & I 3 & & $4 \mathrm{I}$ & 24.18 & 36 & 43 & 9.5 & 0.059070 & 0.028316 \\
\hline Sept. & $\mathbf{I}$ & & 9 & 9.77 & 10 & 55 & $33 \cdot I$ & & & & $x_{4}$ & & 47 & 30.01 & 37 & 0 & 48.6 & & \\
\hline & 2 & & I 3 & 2.92 & I I & 39 & 29.8 & & & & I 5 & & 5.3 & $3^{6.4} \mathrm{I}$ & & I 7 & 9.5 & & \\
\hline & 3 & & r 6 & $5^{8.95}$ & I 2 & 23 & $35 \cdot 3$ & 9.987802 & 0.027928 & & I 6 & 17 & 59 & 43.10 & & $3^{2}$ & I 2.0 & & \\
\hline & 4 & & 20 & 57.93 & 13 & 7 & 47.9 & & & & 17 & 18 & 5 & 49.78 & & 45 & 56.3 & 0.070248 & 0.037469 \\
\hline & 5 & & 24 & 59.92 & 13 & 52 & 6.0 & & & & I 8 & & I I & 56.15 & 37 & $5^{8}$ & 22.6 & & \\
\hline & 6 & & 29 & 5.01 & I 4 & $3^{6}$ & $27 \cdot 7$ & & & & 19 & & 18 & 1.93 & 38 & 9 & $3 \mathrm{r} .5$ & & \\
\hline & 7 & & 33 & 13.26 & I 5 & 20 & $5 \mathrm{I.2}$ & $9.98954 \mathrm{I}$ & 0.02 I 3 I 5 & & 20 & & 24 & 6.82 & & I 9 & 23.4 & & \\
\hline & 8 & & 37 & 24.74 & I 6 & 5 & I 4.5 & & & & $2 \mathrm{I}$ & & 30 & I 0.53 & & 27 & 59.2 & 0.081648 & 0.048037 \\
\hline & 9 & & $4 \mathrm{I}$ & $39 \cdot 5^{2}$ & I 6 & 49 & 35.6 & & & & 22 & & $3^{6}$ & I 2.79 & & 35 & I 9.8 & & \\
\hline & 10 & & 45 & 57.66 & I 7 & 33 & $52 \cdot 5$ & & & & 23 & & 42 & I $3 \cdot 3.3$ & & $4 \mathrm{I}$ & 26.4 & & \\
\hline & $1 \pm$ & & 50 & I 9.24 & I 8 & I 8 & 3.0 & 9.992795 & $0.0 \mathrm{I} 580 \mathrm{I}$ & & $24^{\circ}$ & & 48 & I I. 88 & & 46 & 20.2 & & \\
\hline & I 2 & & 54 & $44 \cdot 32$ & I 9 & 2 & 4.9 & & & & 25 & I 8 & 54 & 8. I 9 & & $5^{\circ}$ & 2.8 & 0.093 I 78 & 0.059866 \\
\hline & 13 & I 4 & 59 & 12.95 & I 9 & 45 & 55.8 & & & & 26 & I9 & 0 & 2.02 & & 52 & 35.7 & & \\
\hline & $I_{4}$ & I 5 & 3 & 45.19 & 20 & 29 & $33 \cdot 3$ & & & & 27 & & 5 & 53.16 & & 54 & 0.5 & & \\
\hline & I 5 & & 8 & 21.10 & 2 I & I 2 & 54.8 & $9.99748 \mathrm{I}$ & 0.011575 & & 28 & & I 1 & $4 \mathrm{I} \cdot 39$ & & 54 & I 8.9 & & \\
\hline & r 6 & & I 3 & 0.72 & $2 \mathrm{I}$ & 55 & 57.9 & & & & 29 & & I 7 & $26.5 \mathrm{I}$ & & 53 & 32.8 & 0.104759 & 0.072789 \\
\hline & I 7 & & I 7 & 44.08 & 22 & $3^{8}$ & 39.9 & & & & $3^{\circ}$ & & 23 & $8.3^{6}$ & & $5 \mathrm{I}$ & 44.0 & & \\
\hline & I 8 & & 22 & $3 x .23$ & 23 & 20 & 58.2 & & & & $3 I$ & & 28 & 46.76 & & 48 & $54 \cdot 5$ & & \\
\hline & I 9 & & 27 & 22.20 & 24 & 2 & 50.2 & 0.003485 & 0.008813 & Nov. & $\mathbf{I}$ & & 34 & $2 x \cdot 5^{6}$ & & 45 & 6.2 & & \\
\hline & 20 & & $3^{2}$ & 17.02 & 24 & 44 & I $3 \cdot I$ & & & & 2 & & 39 & 52.63 & & 40 & $2 \mathrm{I} .2$ & o. I I 6332 & 0.086637 \\
\hline & $2 \mathrm{I}$ & & 37 & I $5.7 \mathrm{I}$ & 25 & 25 & $4 \cdot \mathrm{I}$ & & & & 3 & & 45 & 19.83 & & 34 & $4 \mathrm{I} \cdot 5$ & & \\
\hline & 22 & & $4^{2}$ & I 8.28 & 26 & 5 & $20.6^{\circ}$ & & & & 4 & & $5^{\circ}$ & 43.06 & & 28 & $9 \cdot 3$ & & \\
\hline & 23 & & 47 & 24.73 & 26 & 44 & 59.9 & $0.01067 \mathrm{I}$ & 0.007663 & & 5 & I 9 & 56 & 2.22 & & 20 & 46.6 & & \\
\hline & 24 & & $5^{2}$ & 35.07 & 27 & 23 & 59.2 & & & & 6 & 20 & I & 17.23 & & I 2 & $35 \cdot 5$ & $0.12784 \mathrm{I}$ & 0.101237 \\
\hline & 25 & I 5 & 57 & 49.27 & 28 & 2 & I 5.8 & & & & 7 & & 6 & 28.02 & $3^{8}$ & 3 & $3^{8.2}$ & & \\
\hline & 26 & I 6 & 3 & $7 \cdot 3 \mathrm{I}$ & 28 & 39 & 46.9 & & & & 8 & & I I & 34.53 & 37 & 53 & 56.8 & & \\
\hline & 27 & & 8 & 29.15 & 29 & I 6 & 29.8 & 0.018892 & 0.008243 & & 9 & & I 6 & $3^{6.72}$ & & 43 & $33 \cdot 4$ & & \\
\hline & 28 & & I 3 & 54.73 & 29 & 22 & 21.9 & & & & 10 & & $2 \mathrm{I}$ & 34.55 & & 32 & 30.1 & 0.1 39247 & 0.116433 \\
\hline & 29 & & 19 & 23.96 & 30 & 27 & 20.5 & & & & I I & & 26 & 28.00 & & 20 & 49.0 & & \\
\hline & 30 & & 24 & $5^{6.78}$ & $3^{1}$ & I & 23.2 & & & & I 2 & & $3 r$ & 17.06 & 37 & 8 & 31.9 & & \\
\hline kt. & I & & 30 & 33.09 & $3 \mathrm{I}$ & 34 & $27 \cdot 4$ & 0.027993 & $0.0106 I_{4}$ & & I 3 & & 36 & 1.73 & $3^{6}$ & 55 & 40.6 & & \\
\hline & 2 & & 36 & I 2.77 & $3^{2}$ & 6 & 30.8 & & & & I 4 & & 40 & 42.03 & & 42 & 17.0 & 0.150513 & 0.132073 \\
\hline & 3 & I 6 & $4 \mathrm{I}$ & 55.69 & $3^{2}$ & 37 & 31.8 & & & & I 5 & 20 & 45 & 17.98 & $3^{6}$ & 28 & 22.9 & & \\
\hline
\end{tabular}

Graz, Sternwarte der Universität, 19 × 5 April 7 .

K. Hillebrand.

\section{Photographic observation of comet $1913 \mathrm{c}$ (Neujmin) at Winchester.}

I 9 I 3 Sept. 30 I $2^{\mathrm{h}} 45^{\mathrm{m}} \circ$ Gr. m. t. I9 $3.0: 23^{\mathrm{h}} 35^{\mathrm{m}} 33^{\mathrm{s}}+8^{\circ} 55^{\prime} \cdot 7$. Mag. I 3.o. $\quad$ F. H. Metcalf.

Anzeige. Vom Generalregister der Bände I 5I-I80 liegt der erste Teil, Namen- und Sachregister, I 2 Bogen umfassend, fertig gedruckt vor. Da der Druck der Spezialregister nicht alsbald erfolgen kann und es vielfach erwünscht sein dürfte, das Namen- und Sachregister schon jetzt benutzen zu können, bin ich bereit, diesen ersten Teil des Registers auf Wunsch schon jetzt zu liefern gegen Anzahlung von 12 Mark auf den Preis des ganzen Registers, zu dessen Abnahme nach Fertigstellung der Bezieher verpflichtet sein würde.

Inhalt zu Nr. 48Io. F. Hayn. Gestalt, Größe und gegenseitige Lage von Sonne und Mond. I85. - $K$. Hillebrand. Ephemeride des periodischen Kometen Winnecke für die Erscheinung 1915. 207. - F. H. Metcalf. Photographic observation of comet I913c (Neujmin) at Winchester. 207. - Anzeige. .207. 